\title{
Non-wandering Operator in Bargmann Space
}

\author{
Minggang Wang (Corresponding author) \\ Mathematics Department of Taizhou College, Nanjing Normal University \\ Taizhou 225300, China \\ E-mail: magic821204@sina.com \\ Hua Xu \\ Mathematics Department of Taizhou College, Nanjing Normal University \\ Taizhou 225300, China \\ E-mail:xh0805@sina.com
}

\begin{abstract}
In this paper the Bargmann space is denoted by $F$. This space's roots can be found in mathematical problem of relativistic physics or in quantum optics. In physics the Bargmann space contains the canonical coherent states, so it is the main tool for studying the bosonic coherent state theory of radiation field and for other application .This paper deals with the unilateral backward shift operator $T$ on a Bargmann space $F$. We provide a sufficient condition for an unbounded operator to be non-wandering operator, and then apply the condition to give a necessary and sufficient condition in order that $T$ be a non-wandering operator.
\end{abstract}

Keywords: Non-wandering operator, Unbounded operator, Bargmann space, Hypercyclic operator

\section{Introduction}

It is well known that linear operators in finite-dimensional linear spaces can't be chaotic but the nonlinear operator may be. Only in infinite-dimensional linear spaces can linear operators have chaotic properties. This has attracted widely attention (Godefroy G, 1991; Lixin Tian, 2005; Jiangbo Zhou, 2001; Shaoguang Shi, 2006; Lixin Tian, 2006). Lixin Tian and other researchers introduced non-wandering operators in infinite-dimensional Banach space, which are the generalization of Axiom A dynamic system but different from it. They are new linear chaotic operators and relative to hypercyclic operators, but different from them (Lixin Tian, 2005). In recent years, Jiangbo Zhou discussed the hereditayily hypercyclic decomposition of non-wandering operators in infinite dimensional Frechet space (Jiangbo Zhou, 2001); Shaoguang Shi obtained non-wandering operator sequences on Banach space (Shaoguang Shi, 2006) ; Lihong Ren studied n-multiple non-wandering operator (Lixin Tian, 2006); Minggang Wang studied the pseudo orbit tracing property of invertible nonwandering operator (Lixin Tian, 2007) and Non-wandering Property of Differentiation Operator(Minggang Wang, 2008).

In this paper the Bargmann space is denoted by $F$. This space have been studied by many authors(I.E. Segal, 1963; J.R. Klauder, 1968; J.R. Klauder, 1985; H.Emamirad, 1997). Bargmann space's roots can be found in mathematical problem of relativistic physics (I.E. Segal, 1963) or in quantum optics (J.R. Klauder, 1968). In physics the Bargmann space contains the canonical coherent states, so it is the main tool for studying the bosonic coherent state theory of radiation field (J.R. Klauder, 1985) and for other application (H.Emamirad, 1997).

In finite-dimensional separable Banach space, for the bounded linear operators, Lixin Tian and other researchers have given the definition of non-wandering operator (Lixin Tian, 2005). However, this definition is restricted for the bounded linear operators. In this paper, we consider the non-wandering property of the unbounded operators. Let $T$ be an unbounded operator on a separable infinite dimensional Banach space $X$. It may happen that a vector $x$ is in the domain of $T$, but $T x$ fails to be in the domain of $T$. For this reason, in order to consider the non-wandering property of the unbounded operator, we should firstly suppose that if $x$ in the domain of $T$ then for every integer $n \geq 1$ the vector $T^{n} x$ is in the domain of $T$.

On the basis of the above research, in this paper, we first provide a sufficient condition for an unbounded operator to be non-wandering operator(see Theorem 1), and then apply the condition to give a necessary and sufficient condition in order that $T$ be a non-wandering operator. (see Theorem 2)

\section{Basic notation and definitions}

Definition 2.1(Lixin Tian, 2005) Let $(X,\|\cdot\|)$ be an infinite dimensional separable Banachspace. Suppose $T \in L(X)$

(1) Assume that there exists a closed subspace $E \subset X$, which has hyperbolic structure: $E=E^{u} \oplus E^{s}, T E^{u}=E^{u}, T E^{s}=$ $E^{s}$, where $E^{u}, E^{s}$ are closed subspaces. In addition, there exists constants $\tau(0<\tau<1)$ and $C>0$, such that for any $\xi \in E^{u}, k \in N,\left\|T^{k} \xi\right\| \geq C \tau^{-k}\|\xi\|$, and for any $\eta \in E^{s}, k \in N,\left\|T^{k} \eta\right\| \leq C \tau^{k}\|\eta\|$; 
(2) Assume also that $\operatorname{Per}(T)$ is dense in $E$. Then $T$ is said to be a non-wandering operator relative to $E$.

definition 2.2 Suppose $T \in L(X)$ and $\left\{e_{i}\right\}_{1}^{\infty}$ is a basis in $X$, then $T$ is called a unilateral backward shift operator relative to $\left\{e_{i}\right\}_{1}^{\infty}$ if $T e_{n}=e_{n-1}(n>1)$ and $T e_{1}=0$.

\section{Main results}

Theorem $1 \operatorname{Let}(X,\|\cdot\|)$ be an infinite dimensional separable Banachspace.Tis an unbounded operator, if for $\forall n \geq 1, T^{n}$ is the closed operator and $T$ satisfy (1) there exists a closed subspace $E \subset X$, which has hyperbolic structure; (2) $P e r(T)$ is dense in $E$. Then $T$ is a non-wandering operator relative to $E$.

Proof By the Closed Graph Theorem, we can easily obtain this result.

Remark In fact, from Theorem 1, if an unbounded operator $T$ has non-wandering property, then $T$ need to satisfy: (1) $T^{n}$ is the closed operator, $\forall n \geq 1$; (2) there exists a closed subspace $E \subset X$, which has hyperbolic structure relative to $T$; (3) $\overline{\operatorname{Per}(T)}=E$

In the following, we will apply Theorem 1 to the unilateral backward shift operator $T$ on a Bargmann space $F$.

\subsection{Non-wandering operator in Bargmann space}

Let $\left\{w_{n}\right\}_{n \in N}$ be an arbitrary weight sequence, we define the iterated unbounded back-ward shift $T^{n}$ in Bargmann space by

$$
T^{n}\left(\sum_{k \geq 0} C_{k} \frac{x^{k}}{\sqrt{k !}}\right)=\sum_{k \geq 0}\left(\prod_{j=k}^{n+k-1} w_{j}\right) C_{n+k} \frac{x^{k}}{\sqrt{k !}}
$$

with its domain in $F$, and we define

$$
D\left(T^{n}\right)=\left\{f(x)=\left.\sum_{k \geq 0} C_{k} \frac{x^{k}}{\sqrt{k !}}\left|\sum_{k \geq 0}\right| C_{k}\right|^{2}<\infty ; \sum_{k \geq 0}\left|\prod_{j=k}^{m+k-1} w_{j}\right|^{2}\left|C_{k+m}\right|^{2}<\infty\right\}
$$

for all $m \in N, 1 \leq m \leq n$.

Theorem 2 A linear unbounded backward shift operator $T: F \rightarrow F$ is non-wandering operator if the positive series

$$
\sum_{n=0}^{\infty} \prod_{j=0}^{n-1} \frac{1}{\left|w_{j}\right|^{2}}
$$

converges.

Proof From Theorem 1, we need three steps to proof the theorem:

Firstly, we proof that for $\forall n \in N, T^{n}$ is closed.

By the definition, we choose $\left\{f_{j}\right\} \in D\left(T^{n}\right)$, since $F$ is a Hilber space, then $\left\{f_{j}\right\} \rightarrow f_{0}$ in $F$, so $f_{j}(x)=\sum_{k \geq 0} C_{k, j} \frac{x^{k}}{\sqrt{k !}} \quad \rightarrow$ $f_{0}(x)=\sum_{k \geq 0} C_{k}^{0} \frac{x^{k}}{\sqrt{k !}} \quad j \rightarrow \infty$

$$
\Rightarrow\left(\prod_{j=k}^{n+k-1} w_{j}\right) C_{n+k, j} \rightarrow\left(\prod_{j=k}^{n+k-1} w_{j}\right) C_{n+k}^{0}
$$

Let $T^{n} f_{j} \rightarrow g_{0}$, then $g_{0}(x)=\sum_{k \geq 0} \xi_{k}^{0} \frac{x^{k}}{\sqrt{k !}}$

We can conclude that

$$
\xi_{k}^{0}=\left(\prod_{j=k}^{n+k-1} w_{j}\right) C_{n+k}^{0}
$$

This proves that $f_{0} \in D\left(T^{n}\right)$ and $T^{n} f_{0}=g_{0}$.

(2)Since the positive series $\sum_{n=0}^{\infty} \prod_{j=0}^{n-1} \frac{1}{\left|w_{j}\right|^{2}}$ converges, then $\prod_{j=0}^{n-1} \frac{1}{\left|w_{j}\right|^{2}} \rightarrow 0$, that is, $\prod_{j=0}^{n} w_{j} \rightarrow \infty \quad n \rightarrow \infty$

We choose $w_{j}$ is an increasing sequence. 
For $\forall \lambda \in C$, let $f_{\lambda}(x)=\sum_{k \geq 0}\left(\prod_{j=0}^{k-1} \frac{\lambda}{w_{j}}\right) \frac{x^{k}}{\sqrt{k !}}$, then for $\forall 0<v<1$ and $n \in N$ large enough, we have $|\lambda| \leq v\left|w_{n}\right|$, so

$$
\begin{gathered}
\sum_{k \geq 0} \prod_{j=0}^{k-1}\left|\frac{\lambda}{w_{j}}\right|^{2}=\sum_{k=0}^{n} \prod_{j=0}^{k-1}\left|\frac{\lambda}{w_{j}}\right|^{2}+\sum_{k=n+1}^{\infty} \prod_{j=0}^{k-1}\left|\frac{\lambda}{w_{j}}\right|^{2} \\
\leq \sum_{k=0}^{n} \prod_{j=0}^{k-1}\left|\frac{\lambda}{w_{j}}\right|^{2}+\left(\frac{1}{1-v}\right) \prod_{j=0}^{n}\left|\frac{\lambda}{w_{j}}\right|^{2}<\infty
\end{gathered}
$$

Thus we get $f_{\lambda}(x) \in F$ and

$$
T f_{\lambda}(x)=\sum_{k \geq 0} w_{k}\left(\prod_{j=0}^{k} \frac{\lambda}{w_{j}}\right) \frac{x^{k}}{\sqrt{k !}}=\lambda \sum_{k \geq 0}\left(\prod_{j=0}^{k-1} \frac{\lambda}{w_{j}}\right) \frac{x^{k}}{\sqrt{k !}}=\lambda f_{\lambda}(x)
$$

Therefore, $f_{\lambda}(x)$ is the eigenvector corresponding to the eigenvalue $\lambda$, furthermore, by the arbitrarily of $\lambda$, we can construct the sets:

$$
V_{1}=\left\{\lambda: \quad|\lambda|>1 \quad \lambda \in \delta_{P}(T)\right\}
$$

$V_{2}=\left\{\lambda: \quad 0<|\lambda|<1 \quad \lambda \in \delta_{P}(T)\right\}$, where $\delta_{p}(T)$ is the spectrum of $T$.

Let $E^{u}=\overline{\operatorname{span}\left\{f_{\lambda} ; \lambda \in V_{1}\right\}}$ for $\forall \xi \in E^{u}$, then $\xi=\sum_{i=1}^{\infty} \alpha_{i} f_{\lambda_{i}}=\sum_{i=1}^{\infty} \alpha_{i} \sum_{k \geq 0}\left(\prod_{j=0}^{k-1} \frac{\lambda_{i}}{w_{j}}\right) \frac{x^{k}}{\sqrt{k !}}$ and for $\forall k \in N$, we have

$$
\begin{aligned}
\left\|T^{k}(\xi)\right\|= & \left\|T^{k}\left(\sum_{i=1}^{\infty} \alpha_{i} f_{\lambda_{i}}\right)\right\|=\left\|\sum_{i=1}^{\infty} \alpha_{i} \lambda_{i}^{k} \sum_{k \geq 0}\left(\prod_{j=0}^{k-1} \frac{\lambda_{i}}{w_{j}}\right) \frac{x^{k}}{\sqrt{k !}}\right\| \\
& \geq \mu^{k}\left|\sum_{i=1}^{\infty} \sum_{k \geq 0}\left(\prod_{j=0}^{k-1} \frac{\lambda_{i}}{w_{j}}\right) \frac{x^{k}}{\sqrt{k !}}\right|=\mu^{k}|\xi|
\end{aligned}
$$

where $\mu=\min \left\{\left|\lambda_{i}\right| \quad \lambda_{i} \in V_{1}\right\}>1$. Let $\tau=\frac{1}{\mu}$, then we can easily get $0<\tau<1$. So by (1) we have $\left\|T^{k}(\xi)\right\|=$ $\left\|T^{k}\left(\sum_{i=1}^{\infty} \alpha_{i} f_{\lambda_{i}}\right)\right\|=\left\|\sum_{i=1}^{\infty} \alpha_{i} \lambda_{i}^{k} f_{\lambda_{i}}\right\| \geq \tau^{-k}|\xi|$.

Next, we will prove $E^{u}$ is the invariant subspace of $T$.

Since for $\forall \xi \in E^{u}$, then $\xi=\sum_{i=1}^{\infty} \alpha_{i} f_{\lambda_{i}}=T \sum_{i=1}^{\infty} \frac{\alpha_{i}}{\lambda_{i}} f_{\lambda_{i}}=T E^{u} \Rightarrow \xi \in T E^{u}$, so $E^{u} \subset T E^{u}$. In the other hand, for $\forall \eta \in T E^{u}$, then there exists $\varphi \in E^{u} \Rightarrow \varphi=\sum_{i=1}^{\infty} \beta_{i} f_{\lambda_{i}}$, such that, $\eta=T \varphi=\sum_{i=1}^{\infty} \lambda_{i} \beta_{i} f_{\lambda_{i}} \in E^{u}$. So we can get $T E^{u} \subset E^{u}$, therefore, $T E^{u}=E^{u}$.

Similarly, let $E^{s}=\overline{\operatorname{span}\left\{f_{\lambda} ; \lambda \in V_{2}\right\}}$, then $T E^{s}=E^{s}$, for $\forall \eta \in E^{s}$, we have $\eta=\sum_{i=1}^{\infty} \beta_{i} f_{\lambda_{i}}=\sum_{i=1}^{\infty} \beta_{i} \sum_{k \geq 0}\left(\prod_{j=0}^{k-1} \frac{\lambda}{w_{j}}\right) \frac{x^{k}}{\sqrt{k !}}$, so for $\forall k \in N,\left\|T^{k}(\eta)\right\|=\left\|T^{k}\left(\sum_{i=1}^{\infty} \beta_{i} f_{\lambda_{i}}\right)\right\|=\left\|\sum_{i=1}^{\infty} \beta_{i} \lambda_{i}^{k} f_{\lambda_{i}}\right\| \leq \tau^{k}|\eta|$ where $0<\tau=\max \quad\left\{\left|\lambda_{i}\right| \quad \lambda_{i} \in V_{2}\right\}<1$ and $T E^{s}=E^{s}$

Let $E=E^{u} \oplus E^{s}$, then we can easily get $E$ has hyperbolic structure.

(3) From the definition, we have $T^{n}\left(\sum_{k \geq 0} C_{k} \frac{x^{k}}{\sqrt{k !}}\right)=\sum_{k \geq 0}\left(\prod_{j=k}^{n+k-1} w_{j}\right) C_{n+k} \frac{x^{k}}{\sqrt{k !}}$, if $T$ has the $N$-periodic point, then we have $\left(\prod_{j=k}^{n+k-1} w_{j}\right) C_{n+k}=C_{k}, \forall k \geq 0$, so for $\forall l=0,1 \cdots n-1, k \geq 1$, we have $C_{k n+l}=\left(\prod_{j=l}^{k n+l-1} \frac{1}{w_{j}}\right) C_{l}$, thus for $\forall v \geq 0 \quad n \geq v, n \in$ $N$, we can construct

$$
g_{v, n}(x)=\frac{x^{v}}{\sqrt{v !}}+\sum_{k=1}^{\infty}\left(\prod_{j=v}^{k n+v-1} \frac{1}{w_{j}}\right) \frac{x^{k n+v}}{\sqrt{(k n+v) !}}
$$

Since the series $\sum_{n=0}^{\infty} \prod_{j=0}^{n-1} \frac{1}{\left|w_{j}\right|^{2}}$ converges, then we have $\sum_{k=1}^{\infty} \prod_{j=v}^{k n+v-1} \frac{1}{w_{j}}<\infty$, so, we get $g_{v, n} \in F$ and 


$$
T^{N} g_{v, n}(x)=T^{N}\left(\frac{x^{v}}{\sqrt{v !}}+\sum_{k=1}^{\infty}\left(\prod_{j=v}^{k n+v-1} \frac{1}{w_{j}}\right) \frac{x^{k n+v}}{\sqrt{(k n+v) !}}\right)=\frac{x^{v}}{\sqrt{v !}}+\sum_{k=1}^{\infty}\left(\prod_{j=v}^{k n+v-1} \frac{1}{w_{j}}\right) \frac{x^{k n+v}}{\sqrt{(k n+v) !}} .
$$

Thus $g_{v, n}(x)$ is the $N$-periodic point of $T$.

Let $E_{0}=\operatorname{span}\left\{g_{v, n}(x)\right\}$, in the following we will prove that $E_{0}$ is dense on $F$.

Since for $\forall f(x) \in F$, let $f(x)=\sum_{v=0}^{m} C_{v} \frac{x^{v}}{\sqrt{v !}}$, by the definition of Bargmann space, we have

$$
\left|C_{v} \prod_{j=0}^{v-1} w_{j}\right|<\infty
$$

Suppose $\left|C_{v} \prod_{j=0}^{v-1} w_{j}\right|<1$, then there exists $g(x) \in E_{0}$ and

$$
g(x)=\sum_{v=0}^{m} C_{v} g_{v, n}(x)
$$

so that

$$
\begin{gathered}
\|g-f\|=\left\|\sum_{v=0}^{m} C_{v}\left(g_{v, n}(x)-\frac{x^{v}}{\sqrt{v !}}\right)\right\|=\| \sum_{v=0}^{m}\left(C_{v} \prod_{j=0}^{v-1} w_{j}\right) \sum_{k=1}^{\infty}\left(\prod_{j=0}^{k n+v-1} \frac{1}{w_{j}} \frac{x^{k} v+n}{\sqrt{(k n+v) !}} \|\right. \\
\leq \sum_{v=0}^{m} \| \sum_{k=1}^{\infty}\left(\prod_{j=0}^{k n+v-1} \frac{1}{w_{j}} \frac{x^{k} v+n}{\sqrt{(k n+v) !}} \|\right.
\end{gathered}
$$

Furthermore, from the series $\sum_{n=0}^{\infty} \prod_{j=0}^{n-1} \frac{1}{\left|w_{j}\right|^{2}}$ converges, the there exists $n \geq m$ such that for $\forall \varepsilon>0$, we have

$$
\sum_{k \geq n+1}\left(\prod_{j=0}^{k} \frac{1}{w_{j}} \varepsilon_{k} \frac{x^{k}}{\sqrt{k !}} \|<\frac{\varepsilon}{m+1}\right.
$$

where $\varepsilon_{k}$ taking values 0 or 1 , so we can get when $n \geq m$, then $(2)<\varepsilon$.

Therefore, $E_{0}$ is dense on $F$.

Corollary 1 The operator of differentiation $D: f \rightarrow f^{\prime}$ defined on

$$
\delta=\left\{f \in F \mid f^{\prime} \in F\right\}
$$

is the non-wandering operator on $F$.

Proof Since $\frac{x^{k}}{\sqrt{k !}}$ is an orthonormal basis in $F$, then we have

$$
D\left(\frac{x^{k}}{\sqrt{k !}}\right)=k \cdot \frac{x^{k-1}}{\sqrt{k !}}=\sqrt{k} \frac{x^{k-1}}{\sqrt{(k-1) !}}=w_{k-1} \frac{x^{k-1}}{\sqrt{(k-1) !}}
$$

where $w_{k}=\sqrt{k+1}$,

So, we can get the operator of differentiation $D: f \rightarrow f^{\prime}$ is the weighted backward shift operator, therefore,

$$
D\left(\sum_{k \geq 0} C_{k} \frac{x^{k}}{\sqrt{k !}}\right)=\sum_{k \geq 0} w_{k} C_{k+1} \frac{x^{k}}{\sqrt{k !}} \quad, \quad w_{k}=\sqrt{k+1}
$$

so the series

$$
\sum_{n=0}^{\infty} \prod_{j=0}^{n-1} \frac{1}{\left|w_{j}\right|^{2}}=\sum_{n=0}^{\infty} \prod_{k=0}^{n-1} \frac{1}{k+1}
$$

converges in $F$, thus by the Theorem 2, we have $D$ is the non-wandering operator on $F$. 


\section{Conclusion}

In this paper, we first extent the non-wandering operator theory to the unbounded operator. We provide a sufficient condition for an unbounded operator to be non-wandering operator and get differentiation operator on the Bargmann space be a non-wandering operator. Therefore, the non-wandering operator theory has been further improved, but also enriched the research of the chaotic operator and the Hypercyclic operator.

\section{Acknowledgment}

The author would like to express great gratitude to Professor Lixin Tian of Nonlinear Scientific Research Center of Jiangsu University for useful discussions and valuable suggestions.

\section{References}

Godefroy G, Shapiro J H. (1991). Operators with dense, invariant cyclic vector manifolds. J.Funct Anal, 98:229-269.

H. Emamirad, G.S. Heshmati. (2005). Chaotic weighted shifts in Bargmann space. J. Math. Anal. Appl, 308:36-46.

H.Emamirad, R. Holtz. (1997). Vecteurs d'etat coherent et image de la transformee de Husimi C. R. Acad. Sci. Paris., 324:1295-1300.

I.E. Segal. (1963). Mathematical Problems of Relativistic Physics. Amer. Math. Soc, Providence, RI., 31-46.

J. Bés, K.C. Chan, S.M. Seubert. (2001). Chaotic unbounded differentiation operators. Integral Equations Operator Theory, 40:257-267.

J.R. Klauder, B. Skagerstam. (1985). Coherent States, Application in Physics and Mathematical Physics, World Scientific, Singapore. 21:3895-3908.

J.R. Klauder, E. C. Sudarshan. (1968). Fundamentals of Quantum Optics. Benjamin, New York. 318:36-46.

Jiangbo Zhou, Lixin Tian, Dianchen Lu. (2001). The hereditarily decomposition of non-wandering operators in infinite dimensional Frechet space. Journal of Jiangsu University( Natural Science Edition), 22(6):88 - 91.

Lixin Tian, Jiangbo Zhou, Xun Liu, Guangsheng Zhong. (2005). Nonwandering operators in Banach Space.International Journal Of Mathematics and Mathematical Sciences, 24:3895-3908.

Lixin Tian, Lihong Ren. (2006). N-multiple Nonwandering UnilateralWeighted Backward Shift Operators and the Property of Direct Sum Operators in Banach Space. International Journal of Nonlinear Science, 2(2): 104-110.

Lixin Tian, Minggang Wang. (2007). Pseudo orbit tracing property of non-wandering operator. International Journal of Nonlinear Science, 1(3): 3-7.

Minggang Wang. (2008). Non-wandering Property of Differentiation Operator. International Journal of Nonlinear Science, (6):21-28.

Shaoguang Shi, Guangsheng Zhong. (2006). Nonwandering operator sequences in Banach space. International Journal of Nonlinear Science, 1(3):164-171. 